\title{
Temporal Effects of Mechanical Loading on Deformation-Induced Damage in Skeletal Muscle Tissue
}

\author{
S. Loerakker, ${ }^{1}$ A. Stekelenburg,${ }^{2}$ G. J. Strijkers, ${ }^{2}$ J. J. M. Rijpkema, ${ }^{3}$ F. P. T. Bahijens, ${ }^{2}$ \\ D. L. BAder, ${ }^{2,4}$ K. Nicolay, ${ }^{2}$ and C. W. J. Oomens ${ }^{2}$ \\ ${ }^{1}$ Department of Biomedical Engineering, Eindhoven University of Technology, P.O. Box 513, Building W-Hoog 4.108, 5600 \\ MB Eindhoven, The Netherlands; ${ }^{2}$ Department of Biomedical Engineering, Eindhoven University of Technology, P.O. Box 513, \\ 5600 MB Eindhoven, The Netherlands; ${ }^{3}$ Department of Mathematics and Computer Science, Eindhoven University of \\ Technology, P.O. Box 513, 5600 MB Eindhoven, The Netherlands; and ${ }^{4}$ Department of Engineering and IRC in Biomedical \\ Materials, Queen Mary, University of London, Mile End Road, London E14NS, UK
}

(Received 16 November 2009; accepted 4 March 2010; published online 16 March 2010)

Associate Editor Catherine Disselhorst-Klug oversaw the review of this article.

\begin{abstract}
Mechanical loading of soft tissues covering bony prominences can cause skeletal muscle damage, ultimately resulting in a severe pressure ulcer termed deep tissue injury. Recently, by means of an experimental-numerical approach, it was shown that local tissue deformations cause tissue damage once a deformation threshold is exceeded. In the present study, the effects of load exposure time and intermittent load relief on the development of deformationinduced muscle damage were investigated. The data showed that a $2 \mathrm{~h}$ loading period caused more damage than $10 \mathrm{~min}$ loading. Intermittent load reliefs of 2 min during a $2 \mathrm{~h}$ loading period had minimal effect on the evolution of skeletal muscle damage. In addition, a local deformation threshold for damage was found, which was similar for each of the loading regimes applied in this study. For short loading periods, these results imply that local tissue deformations determine whether muscle damage will develop and the exposure time influences the amount of tissue damage. Temporary load reliefs were inefficient in reducing deformation-induced damage, but may still influence the development of ischemia-induced damage during longer loading periods.
\end{abstract}

Keywords-Pressure ulcers, Deep tissue injury, Magnetic resonance imaging, Finite element modeling, Damage threshold.

\section{INTRODUCTION}

Prolonged mechanical loading of soft tissues covering bony prominences, as present when individuals are bedridden or wheelchair-bound, may lead to degeneration of skeletal muscle tissue. This can result

Address correspondence to S. Loerakker, Department of Biomedical Engineering, Eindhoven University of Technology, P.O. Box 513, Building W-Hoog 4.108, 5600 MB Eindhoven, The Netherlands. Electronic mail: s.loerakker@tue.nl in a condition termed pressure-related deep tissue injury (DTI), a severe form of pressure ulcer that initiates in deep tissue layers under an intact skin. ${ }^{2}$ Subsequently, this tissue damage can progress toward the skin and develop into an extensive wound, with a variable prognosis due to complications, such as osteomyelitis, sepsis, and an increased mortality rate. ${ }^{26}$

The etiology of different forms of pressure ulcers is not fully understood. Traditionally, compressioninduced ischemia is considered to represent the primary etiological factor. However, also other damage pathways are involved, such as impaired lymphatic drainage, ${ }^{20}$ ischemia/reperfusion injury, ${ }^{21,27,28}$ and sustained tissue deformation. ${ }^{5,6,11,24}$ Recently, animal experiments of Stekelenburg et al. ${ }^{25}$ showed that $2 \mathrm{~h}$ of continuous muscle compression caused damage in specific regions of the muscle while the complete tissue was ischemic during loading. Finite element simulations of these experiments by Ceelen et al. ${ }^{7}$ demonstrated that these regions of damage coincided with the regions subjected to the largest deformations. In addition, muscle damage was only observed in experiments for which a distinct strain threshold was exceeded. This indicates that when local tissue deformations exceed a critical strain threshold, deformation can play an important role in the etiology of DTI and for a continuous loading period of $2 \mathrm{~h}$, it is more harmful to the tissue than ischemia.

In clinical practice, however, individuals are subjected to a range of loading regimes, associated with repositioning and pressure relief strategies for wheelchair-bound subjects. It is therefore important to investigate how the relationship between deformation and damage depends on the applied loading regime. In 
the present study, two questions were addressed: (1) Do the strain-damage relationship and the critical strain threshold for skeletal muscle tissue depend on the load exposure time? (2) Does intermittent load relief as present during repositioning schemes affect the damage evolution?

\section{MATERIALS AND METHODS}

A combined experimental-numerical approach was adopted to investigate the relationship between muscle deformation and damage, as illustrated in Fig. 1. Animal experiments were performed to monitor the damage evolution in mechanically loaded muscle tissue by means of magnetic resonance imaging (MRI). By simulating these experiments with dedicated finite element (FE) models, the local deformations in the muscle tissue during indentation could be estimated.

\section{Animal Experiments}

A previously developed animal model was used to study the damage evolution in skeletal muscle tissue. $^{7,23,24}$ In this model, 3- to 4-month-old female
Brown-Norway rats were used for which the tibialis anterior (TA) muscle in the left hind limb was mechanically loaded with an indenter. Animals were housed under well-controlled laboratory conditions (12 h light, $12 \mathrm{~h}$ dark cycles) and maintained on standard chow and water ad libitum. Rats were anesthetized with $0.6 \mathrm{~L} / \mathrm{min}$ medical air with $3 \%$ isoflurane for induction and $1-2 \%$ for maintenance. Respiratory rate was monitored and maintained within the physiological range. The animal experiments were approved and supervised by the Animal Care Committee of Maastricht University.

\section{Experimental Protocol}

The experimental set-up and protocol have been described in detail elsewhere. ${ }^{7,23,24}$ To summarize briefly, hairs on the left hind limb of the rat were removed by shaving, after which the left limb was placed in a specially designed mold and fixated with plaster cast. The anesthetized animal was positioned supine in the loading device, consisting of two concentric tubes, as illustrated in Fig. 2. The inner one housed the animal, while the outer tube was used to position the rat in a $6.3 \mathrm{~T}$ MR scanner (Bruker System,
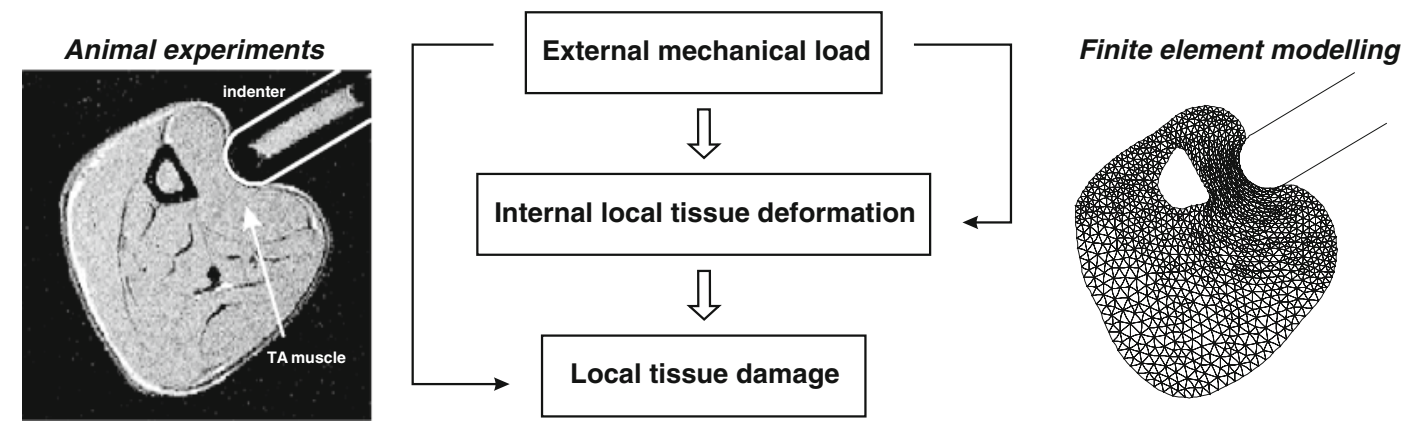

FIGURE 1. Schematic overview of the experimental-numerical approach. Animal experiments were performed in which the damage evolution due to compressive loading was studied with MRI. Dedicated FE models were developed to estimate local tissue deformations during loading. Left: MR image of a cross-section of the lower leg of a rat with an indenter compressing the tibialis anterior (TA) muscle. MRI was also used to detect locations of muscle damage after release of the indenter. Right: Dedicated FE model of the corresponding cross-section of the leg.

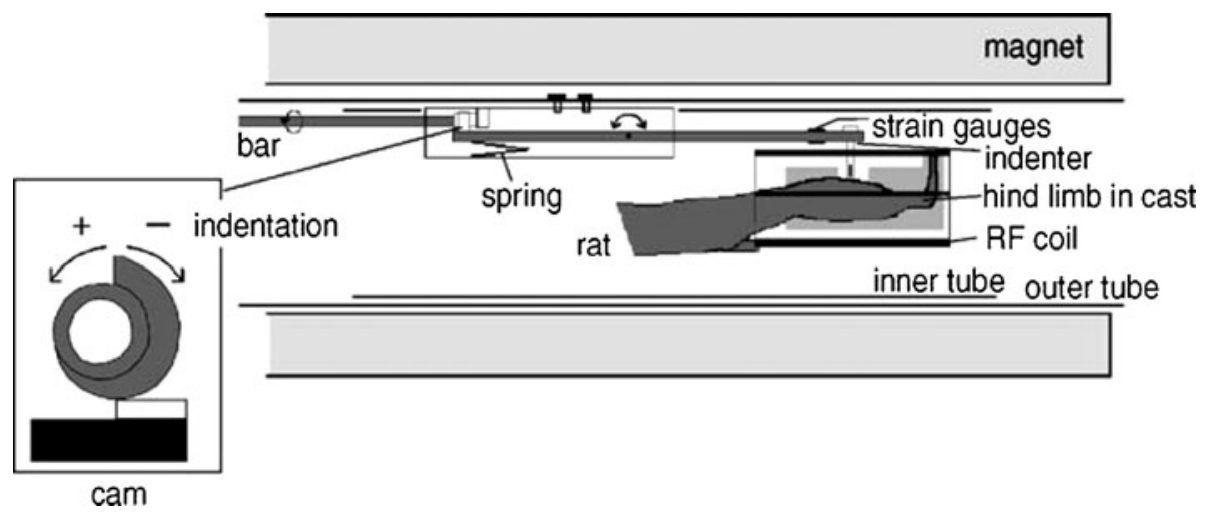

FIGURE 2. Schematic representation of the experimental setup (from Stekelenburg et al. ${ }^{23}$ with permission). 
(a) $1 \times 120 \min (n=11)$

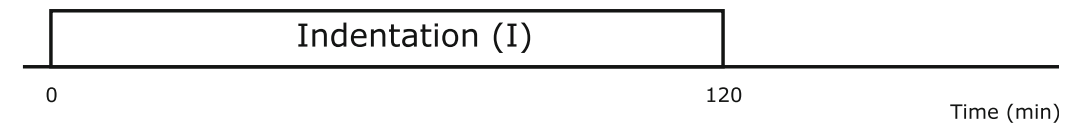

(b) $1 \times 10 \min (n=8)$

\begin{tabular}{llll}
\hline $\mathrm{I}$ & & \\
\hline 0 & 10 & Time (min)
\end{tabular}

(c) $12 \times 10 \min (n=6)$

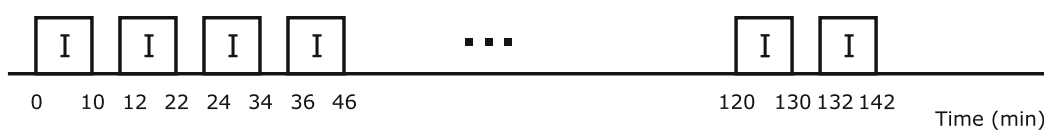

FIGURE 3. Overview of three loading regimes. (a) $2 \mathrm{~h}$ continuous loading of the tibialis anterior (TA) muscle by indentation. ${ }^{7}$ This loading regime was compared with (b) a shorter loading period (10 $\mathrm{min})$ and (c) with $2 \mathrm{~h}$ intermittent loading (12 $\times 10 \mathrm{~min}$ loading with 2 min recovery in between). $n$ denotes the number of animals that were subjected to the different loading regimes.

whorizontal bore, inner diameter $120 \mathrm{~mm}$ ) with a $400 \mathrm{mT} / \mathrm{m}$ gradient coil. The rat was placed on a heating pad to maintain body temperature within physiological values. The left foot was fixed with a special holder, and a birdcage radio-frequency coil was placed around the limb in a fixed position. A hole in the plaster cast enabled the application of a plastic cylindrical indenter (diameter $3 \mathrm{~mm}$, length $6 \mathrm{~mm}$, attached to a rod) to the TA muscle. The cylinder compressed the tissue with its long axis aligned with the longitudinal axis of the limb, such that a distance of $6 \mathrm{~mm}$ underneath the indenter was subjected to approximately uniform indentation in the transverse plane.

The damage evolution in the TA muscle was investigated for three distinct loading regimes, as indicated in Fig. 3. The effect of load exposure time was studied by comparing $2 \mathrm{~h}$ loading (Fig. 3a) with 10 min loading (Fig. 3b). The $2 \mathrm{~h}$ continuous loading regime was also compared with $2 \mathrm{~h}$ of intermittent loading $(12 \times 10 \mathrm{~min}$ loading with 2 min recovery in between, Fig. 3c), to investigate the influence of periodic off-loading on the damage evolution. For the $2 \mathrm{~h}$ continuous loading regime, experimental data $(n=11)$ from Ceelen et al. ${ }^{7}$ were used. For the 10 min loading and $2 \mathrm{~h}$ intermittent loading regimes, 8 and 6 animals were used, respectively.

\section{MR Measurements}

Transversal scout images were obtained to assess the geometry of the rat leg and the angle and initial position of the indenter. Damage in the TA muscle as a result of indentation was assessed $90 \mathrm{~min}$ after the completion of each loading regime with $\mathrm{T}_{2}$-weighted MRI (multi-echo spin echo sequence with slice thickness $=1 \mathrm{~mm}, \quad F O V=25 \times 25 \mathrm{~mm}^{2}$, matrix size $=$ $128 \times 128$ pixels, number of signal averages $=2$, echo time $\mathrm{TE}=10-320 \mathrm{~ms}$, number of echoes $=32$, repetition time $\mathrm{TR}=4 \mathrm{~s}$, fat suppression). To obtain a quantitative $T_{2}$ map, signal intensities (SI) of successive echoes with time (TE) were fitted, on a pixelto-pixel basis, to the equation

$$
S I=A+B e^{-\mathrm{TE} / \mathrm{T}_{2}}
$$

In the $T_{2}$ map before loading, a region of interest in the TA muscle was selected to determine the mean basal $T_{2}$ value and its standard deviation. In the $T_{2}$ map after loading, regions covering at least 3 adjacent pixels with elevated $\mathrm{T}_{2}$ compared with the mean basal value plus 3 times the standard deviation were selected as areas with significantly increased $T_{2}$ values. Elevated $T_{2}$ values have been previously associated with histologically observed muscle damage. ${ }^{24}$

\section{Finite Element Model}

For each animal, dedicated plane stress FE models were developed for 3-4 MR slices underneath the indenter in each experiment, partly based on a previously validated dedicated FE model by Ceelen et al. ${ }^{7,8}$ In this way, the observed geometric differences between rats were accommodated. To create the mesh, transversal scout images of the rat leg before loading were used to detect the outer contours of the leg and the tibia in Matlab (The Mathworks, Inc.), as illustrated in Fig. 4a. The tibia was assumed to be rigid. The muscle tissue was considered incompressible and was modeled as a single-mode hyperelastic Ogden material with strain energy density $W$ :

$$
W=\frac{\mu}{\alpha}\left(\lambda_{1}^{\alpha}+\lambda_{2}^{\alpha}+\lambda_{3}^{\alpha}-3\right)
$$

In experiments of Bosboom et al., ${ }^{4}$ the parameters of the Ogden model for transverse skeletal muscle tissue 

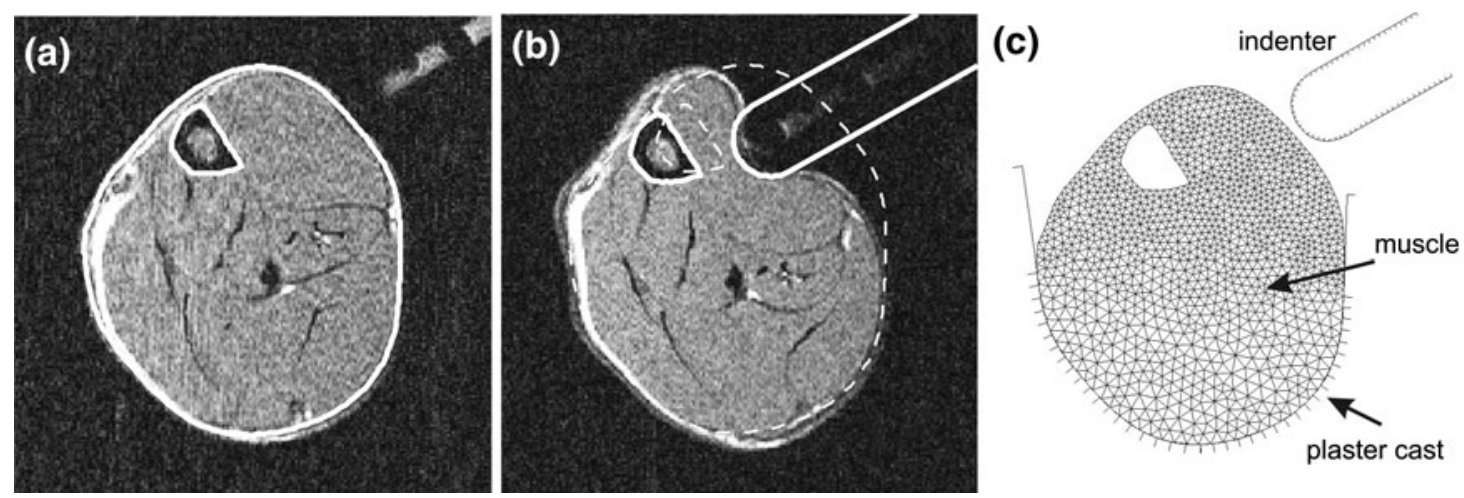

FIGURE 4. (a) Transversal scout image of a cross-section of a rat leg before loading. Contours of leg and tibia were determined (white lines) and used for mesh generation. (b) Scout image of the same rat leg during loading. Position of the tibia during loading and angle and depth of indentation were determined (solid lines) and used for the essential boundary conditions (contours of leg before loading are shown by dashed lines). (c) Resulting FE mesh of the rat leg with indenter and plaster cast modeled as rigid bodies.

were estimated at $\mu=15.6 \mathrm{kPa}$ and $\alpha=21.4$. However, since such a large value for $\alpha$ causes convergence problems in the simulations, $\alpha=5$ was chosen for the present model, and $\mu$ was scaled accordingly $(\mu=3.6 \mathrm{kPa})$ to keep the ratio of $\mu$ and $\alpha$, representing the initial stiffness of the material, equivalent to the one determined by Bosboom et al. ${ }^{4} \lambda_{i}(i=1,2,3)$ are the principal stretch ratios.

From scout images of the deformed leg, the movement of the tibia during indentation, and the angle and depth of indentation were derived (Fig. 4b). In the FE model, the displacements of the tibia and the indenter were prescribed as essential boundary conditions. In the present study, Coulomb friction was assumed between the leg and the indenter, where the friction coefficient was adapted (between 0 and 1) for each FE model to optimize the correspondence of the outer contour of the leg during indentation between experiment and simulation, as determined by visual inspection. The plaster cast surrounding the leg was modeled as a rigid body in free-slip contact with the leg (Fig. 4c). The FE model was implemented in MSC.Marc (MARC Analysis Research Corporation, 2005).

\section{Data Analysis}

To compare local tissue deformations during indentation with damage, the original undeformed $\mathrm{FE}$ mesh was mapped to the situation in the $T_{2}$ map 90 min after unloading (Fig. 5a). ${ }^{7}$ In this quantitative $\mathrm{T}_{2}$ image, a grid (points located at pixel centers with a spacing of $\sim 0.2 \mathrm{~mm}$ ) was superimposed on the TA muscle region, and the grid points at which $T_{2}$ was significantly increased were identified (Fig. 5b). Subsequently, the grid was mapped to the original undeformed FE mesh to obtain a reference configuration in the situation before loading, as shown in Fig. 5c. From this, the nodal displacements of the mesh during indentation, as determined by the FE analysis, were interpolated onto the grid points, to deform the grid to the situation during loading (Fig. 5d). With the configuration of the grid before and during loading, the 2D deformation gradient tensor $\boldsymbol{F}$ during indentation was determined in each grid point, using a secondorder method to compute strains from a discrete set of displacements. ${ }^{12}$ This approach enabled the information on $T_{2}$ increase in the grid points to be compared with the tissue deformations at the same locations. From $\boldsymbol{F}$, the principal stretch ratios $\lambda_{i}(i=1,2,3)$ were determined for each grid point and the strain energy density $W$ was calculated. Thus, in each grid point both the value of $W$ and the presence or absence of a significant increase in $T_{2}$ were determined.

Two analyses were performed to investigate the relationship between deformation and damage (Fig. 6). In the first analysis, global measures of deformation and damage were used to compare the different loading regimes. In the second analysis, deformation and damage were compared at a local level to establish a material property for muscle tissue independent of the specific geometry and deformation pattern of the experiments.

\section{Global Analysis}

As a measure of deformation, the 2D strain energy $E$ needed to deform the TA muscle was calculated for each cross-section by integrating $W$ over the grid (Fig. 6a):

$$
E=\int_{A} W d A=\left(\sum_{i=1}^{N_{\mathrm{gp}}} W_{i}\right) A_{\text {pix }}
$$

where $N_{\mathrm{gp}}$ is the number of grid points, $W_{i}$ is the value of $W$ in grid point $i$, and $A_{\text {pix }}$ is the pixel area. The total 

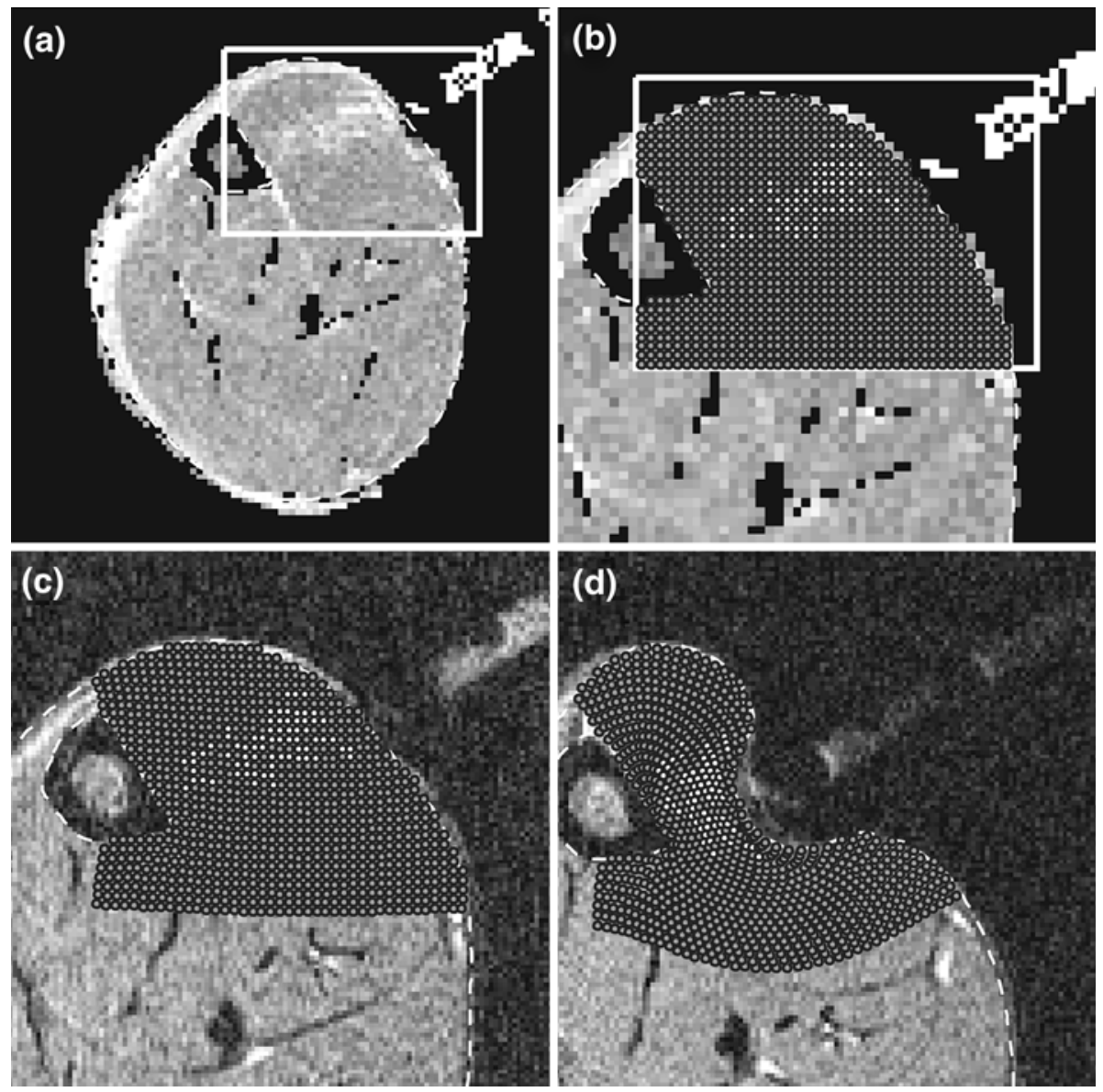

FIGURE 5. (a) $\mathrm{T}_{2}$ map of a rat leg $90 \mathrm{~min}$ after unloading. A region of interest around the TA muscle was selected (white box). (b) A grid was positioned at the pixel centers within this region. Grid points where $T_{2}$ was significantly increased are shown in white. (c) The grid was mapped to the original undeformed FE mesh to obtain a reference configuration. (d) Grid deformed to the situation during loading.

area $A_{d}$ of significantly increased $\mathrm{T}_{2}$ values was determined for each cross-section as a global measure of damage:

$$
A_{d}=N_{d} A_{\text {pix }}
$$

where $N_{d}$ is the number of grid points with an elevated $\mathrm{T}_{2}$. One value of $E$ and $A_{d}$ per experiment was obtained by averaging the values of $E$ and $A_{d}$ over the cross-sections.

\section{Local Analysis}

It was assumed that the local presence of damage in the tissue follows a binomial distribution with a probability $p$ that depends on the local value of $W$. To determine $p$, a smaller area was selected in each grid (red box in Fig. 6b). In this area, approximately 25 regions of $3 \times 3$ grid points $(0.6 \mathrm{~mm} \times 0.6 \mathrm{~mm})$ were defined. The distance between these regions was 1 pixel to minimize the spatial dependency of the results in neighboring regions. For each region, the mean value of $W$ was obtained as a local measure of deformation:

$$
\bar{W}=\frac{1}{9} \sum_{i=1}^{9} W_{i}
$$

The number of grid points $\left(n_{d}\right)$ with elevated $\mathrm{T}_{2}$ in the area was used to estimate the local probability on damage:

$$
p=\frac{1}{9} n_{d}
$$

The relationship between $\bar{W}$ and $p$ was determined by fitting the results of each loading regime to the following logistic regression model ${ }^{16}$ :

$$
\ln \left(\frac{p}{1-p}\right)=b_{1}+b_{2} \bar{W}
$$

where $b_{1}$ and $b_{2}$ are the regression coefficients. To estimate a local deformation threshold for damage, a receiver operating characteristic (ROC) curve was 
(a)

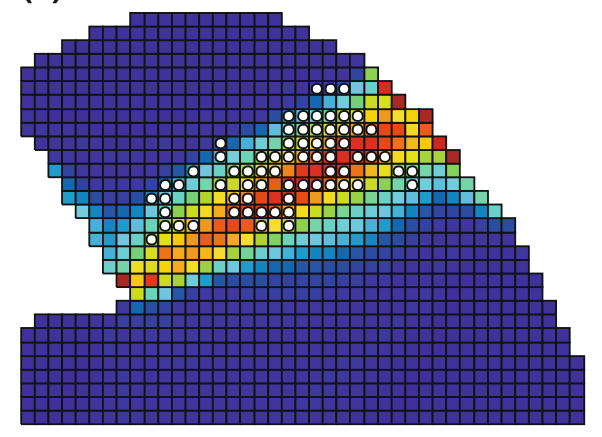

(b)

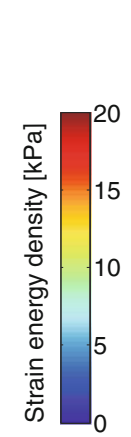

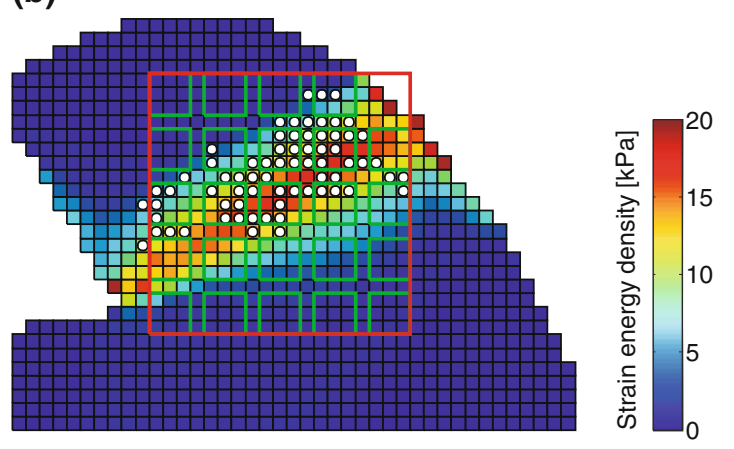

FIGURE 6. Strain energy density in the grid (color) where locations with a significant increase in $\mathrm{T}_{2}$ are indicated by white circles. (a) Global analysis: The strain energy density was integrated over the grid to obtain the 2D strain energy, and the area of elevated $\mathrm{T}_{2}$ values in the grid was used as a measure of damage. (b) Local analysis: $\sim 25$ regions (green) of $3 \times 3$ grid points were positioned in a smaller area (red box). For each region, the mean strain energy density and the fraction of grid points with elevated $T_{2}$ were determined.

determined for each loading regime to determine an optimal cutoff value for $\bar{W}$ to discriminate between deformations with a high or low risk of causing damage. ${ }^{1,22}$ The ROC curves were constructed by plotting the sensitivity, which is the probability of correctly predicting damage in case of damage, vs. $(1-$ specificity), where specificity equals the probability of correctly predicting that there is no damage in case of no damage, for a range of cutoff values for $\bar{W}$. Then, the point on the ROC curve closest to the upper left corner $(0,1)$ of the graph was determined as the optimal cutoff value for balancing the sensitivity and specificity. ${ }^{1}$

\section{RESULTS}

Figure 7 shows the MR images and contours of the accompanying FE models (yellow lines) for a typical experiment for each loading regime. Large differences were evident between the shapes of the legs of the different animals (Figs. 7a, 7e, and 7i), indicating the necessity of developing separate dedicated FE models for each animal. The contours of the FE models of the rat legs during indentation showed a good correspondence with the actual contours of the deformed legs in the MR images (Figs. 7b, 7f, and $7 \mathrm{j}$ ). The $\mathrm{T}_{2}$ maps show that elevated $T_{2}$ values in the TA muscle were concentrated in a narrow region extending from the skin underneath the indenter to the tibia for all three loading regimes (Figs. $7 \mathrm{c}, 7 \mathrm{~g}$, and $7 \mathrm{k}$ ).

The distributions of $W$ in the grid, derived from the FE analyses, are shown in Figs. 7d, 7h, and 71, where the locations with significantly increased $T_{2}$ are indicated by white circles. For all three loading regimes, elevated $T_{2}$ values were mainly present in highly deformed areas. For the $2 \mathrm{~h}$ continuous loading regime, the region of $T_{2}$ increase clearly corresponded to the region of high values of $W$ (Fig. 7d). However, the spatial $T_{2}$ pattern was slightly different from that of the deformation profile. For the other two loading regimes, there was very close correspondence between the regions of $T_{2}$ increase and high deformations (Figs. 7h and 7l).

\section{Global Analysis}

The results of the global comparison of deformation and damage are depicted in Fig. 8. For each experiment, the mean area $A_{d}$ with elevated $\mathrm{T}_{2}$ in the TA muscle is plotted against the mean 2D strain energy $E$ in the muscle during indentation. Figure 8 shows that no $T_{2}$ increase occurred for all three loading regimes when $E$ was less than a threshold zone ranging from $0.8 \times 10^{-4}$ to $1.0 \times 10^{-4} \mathrm{~J} / \mathrm{mm}$. When this threshold was exceeded, $A_{d}$ increased monotonically with $E$. When the two continuous loading regimes are compared, this increase of the affected area with $E$ was larger for $2 \mathrm{~h}$ loading than for $10 \mathrm{~min}$. By contrast, there were little differences in size of the affected region at corresponding $E$ values, when the results of $2 \mathrm{~h}$ continuous loading are compared with $2 \mathrm{~h}$ intermittent loading.

\section{Local Analysis}

Figure 9a shows the probability $p$ on a significant increase in $T_{2}$ value as a function of $\bar{W}$ for each loading regime. For both $10 \mathrm{~min}$ continuous and $2 \mathrm{~h}$ intermittent loading, the probability was approximately zero for $\bar{W}=0$ and increased with $\bar{W}$. However, beyond $\bar{W} \approx 5 \mathrm{kPa}$, the curves of these two loading regimes started to diverge, with $p$ values for $2 \mathrm{~h}$ intermittent loading being consistently higher than 

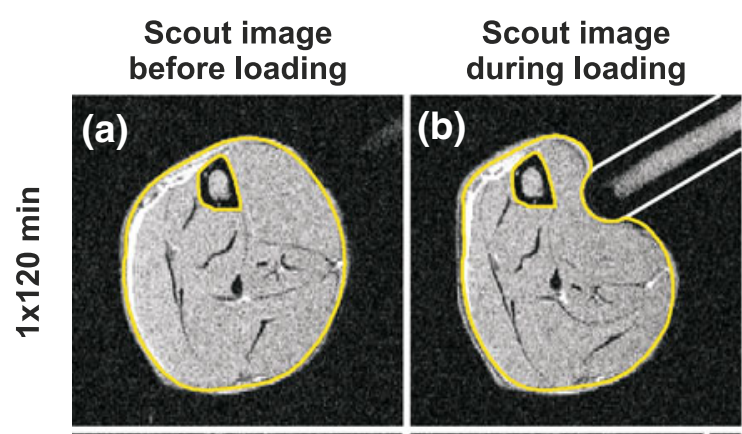

\section{$\mathrm{T}_{2}$ map $90 \mathrm{~min}$ after loading}
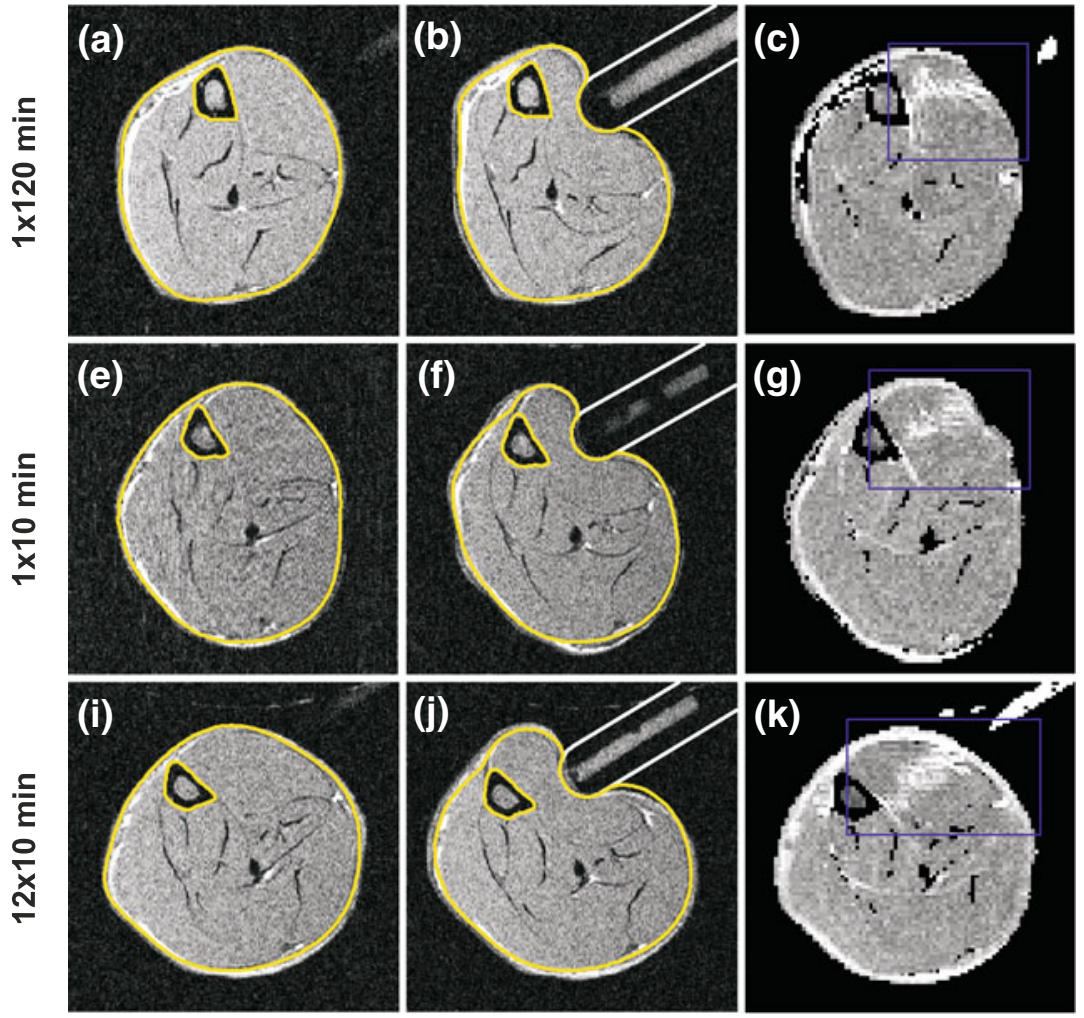

Strain energy density + locations of $\mathrm{T}_{2}$ increase

(d)

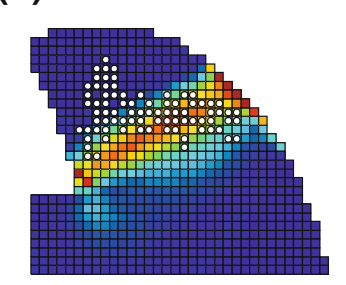

(h)
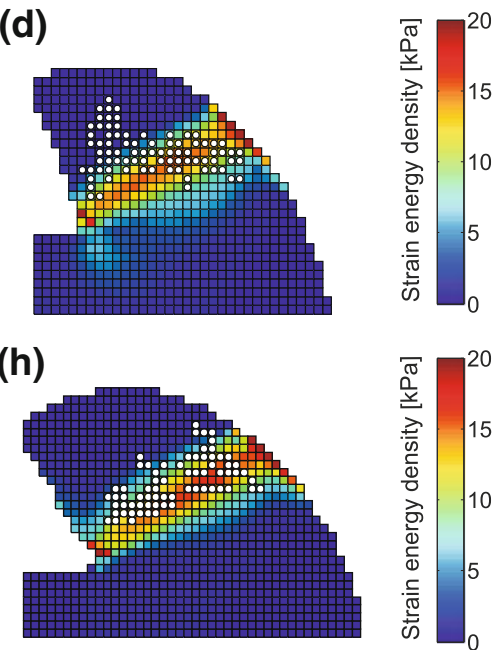

(I)

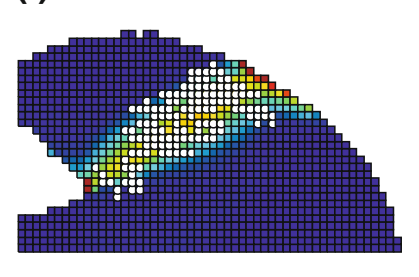

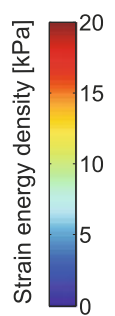

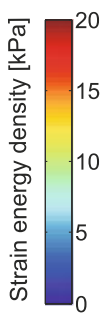

FIGURE 7. Scout images with corresponding FE model contours (yellow), $\mathrm{T}_{2}$ maps with ROI (purple) including the TA muscle, and distributions of $W$ (color) together with locations of $\mathrm{T}_{2}$ increase (white circles) in the ROI for a typical experiment with $2 \mathrm{~h}$ continuous loading (a-d), 10 min loading $(e-h)$, and $2 \mathrm{~h}$ intermittent $(12 \times 10 \mathrm{~min})$ loading (i-l).

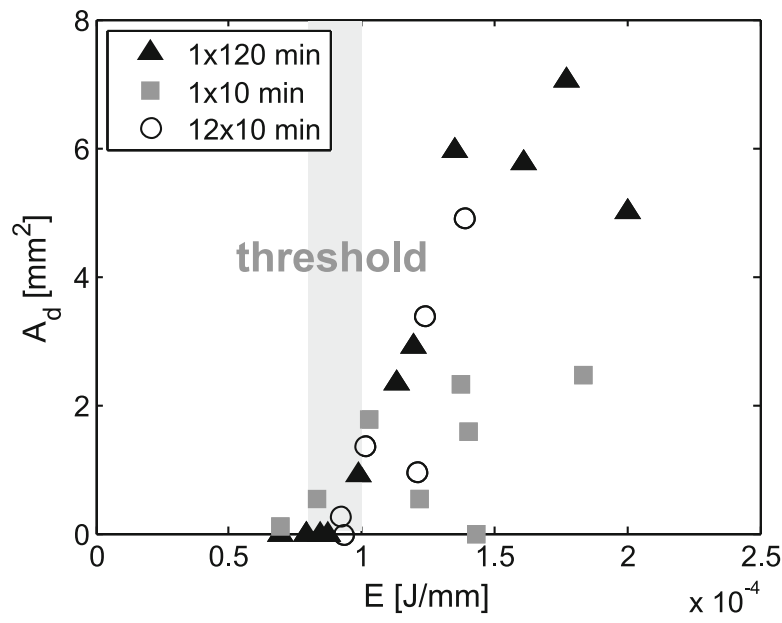

FIGURE 8. Mean area $A_{d}$ with significantly increased $\mathrm{T}_{2}$ values in the TA muscle vs. the mean $2 \mathrm{D}$ strain energy $E$ in the muscle during indentation.

those for 10 min loading. For $2 \mathrm{~h}$ continuous loading, $p$ was slightly larger than zero when $\bar{W}=0$, and increased further with $\bar{W}$. Differences were also evident between the derived probability curves of the $2 \mathrm{~h}$ loading regimes, with a steeper increase of $p$ with $\bar{W}$ for intermittent loading when compared to continuous loading (Fig. 9a).

From the ROC curves, depicted in Fig. 9b, the optimal cutoff values for $\bar{W}$ can be determined to discriminate between high- and low-risk deformations. These values, as indicated in Table 1, reveal a local threshold value of approximately 6-7 $\mathrm{kPa}$ for each of the three loading regimes.

\section{DISCUSSION}

In the present study, the effects of load exposure time and intermittent load relief on the development of deformation-induced skeletal muscle damage were investigated in a rat model, using a combination of MRI techniques and dedicated FE models. The effect of exposure time was investigated by comparing $2 \mathrm{~h}$ and 10 min continuous loading periods. The $2 \mathrm{~h}$ continuous loading period was also compared with a $2 \mathrm{~h}$ intermittent loading protocol to assess the effect of periodic relief of loading. It was found that muscle damage only occurred beyond a deformation threshold, 

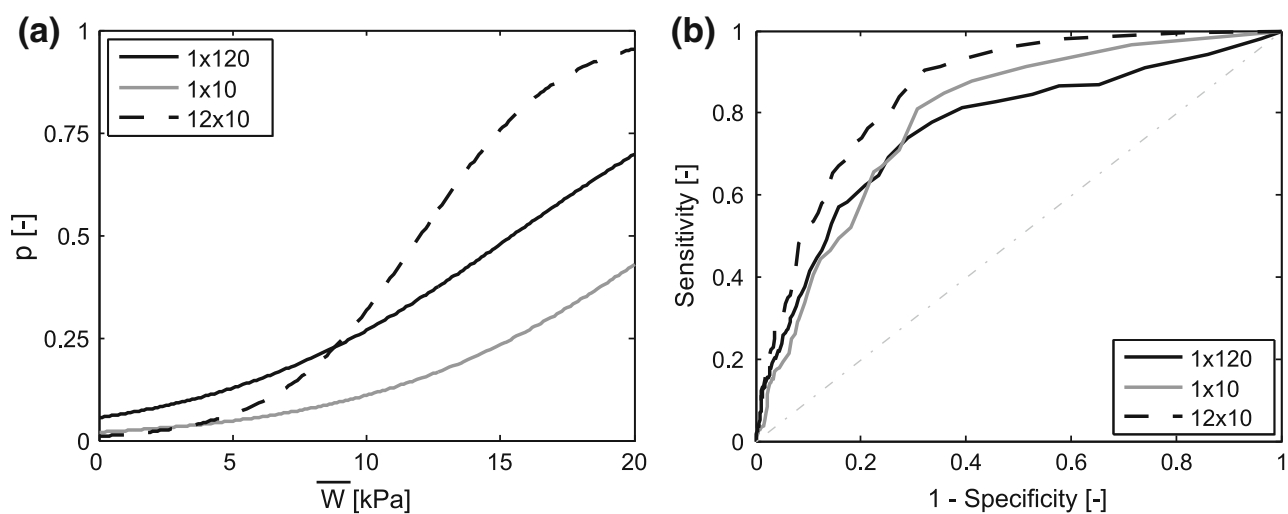

FIGURE 9. (a) Probability $p$ on a significant increase in $T_{2}$ as a function of the mean strain energy density $\bar{W}$. (b) Receiver operating characteristic (ROC) curves for the three loading regimes.

TABLE 1. Parameters of the logistic regression models $\left(b_{1}\right.$ and $\left.b_{2}\right)$ and optimal cutoff values $\left(p_{c}\right.$ and $\left.\bar{W}_{c}\right)$ to discriminate between high- and low-risk deformations, as derived from the ROC curves.

\begin{tabular}{lcccc}
\hline Loading regime & $b_{1}$ & $b_{2}\left(\mathrm{kPa}^{-1}\right)$ & $p_{\mathrm{c}}$ & $\bar{W}_{\mathrm{c}}(\mathrm{kPa})$ \\
\hline $1 \times 120 \mathrm{~min}$ & -2.8 & 0.18 & 0.15 & 6.0 \\
$1 \times 10 \mathrm{~min}$ & -3.9 & 0.18 & 0.07 & 7.2 \\
$12 \times 10 \mathrm{~min}$ & -4.6 & 0.38 & 0.09 & 5.9 \\
\hline
\end{tabular}

$p_{\mathrm{c}}$ is the optimal cutoff probability, $\bar{W}_{\mathrm{c}}$ is the optimal cutoff value for $\bar{W}$.

which was similar for each of the three loading regimes. When the threshold was exceeded, the amount of muscle damage was larger after a $2 \mathrm{~h}$ loading period than after $10 \mathrm{~min}$ of loading. Brief periods of load relief during a $2 \mathrm{~h}$ loading period did not have any significant effect on the damage evolution.

A well-established animal model was used to study the development of deformation-induced muscle damage by means of $\mathrm{T}_{2}$-weighted $\mathrm{MRI}{ }^{23,24} \mathrm{An}$ increase in the transverse relaxation time $T_{2}$ is generally accepted as an indication of tissue damage. ${ }^{10}$ Moreover, in previous studies a good correlation was reported between $\mathrm{T}_{2}$-weighted MRI and skeletal muscle damage assessed histologically.,24 Therefore, locations in the tissue exhibiting a significant increase in $T_{2}$ value after loading were considered to represent areas of tissue damage. However, several pathological mechanisms, e.g. edema, necrosis, and inflammation, may contribute to the $T_{2}$ elevation and can therefore not be distinguished. Additionally, the $T_{2}$ increase observed in this study reflects early cellular damage and swelling, and not an advanced state of damage as present during deep tissue injury. ${ }^{24}$

The internal tissue deformations during loading were simulated by means of dedicated FE models, based on a previously validated FE model of Ceelen et $a l^{7,8}$ The use of dedicated FE models to estimate the internal deformations during loading enabled the comparison of deformation and damage within the same experiment. In addition, this approach could account for the variability in the observed amounts of damage between experiments due to different tissue geometries and deformations. The FE models reported from our previous study showed good agreement with internal muscle deformations as determined from MR-tagging data. ${ }^{8}$ The high level of agreement of the deformed FE model contours with the MR images in that study supported the assumption of a plane stress situation. Furthermore, since displacements were prescribed, the geometry and boundary conditions had a larger influence on the resulting strains than the material properties of the muscle tissue. In the present study, a number of adaptations were implemented in the model to further improve the correspondence between FE model and experiment. A hyperelastic Ogden model was used with a higher order of nonlinearity than the NeoHookean material law that was used in the original FE model of Ceelen et al. ${ }^{8}$ The model parameters were partly based on experiments of Bosboom et al., ${ }^{4}$ but the high order of nonlinearity that was estimated in that study could not be imposed due to convergence problems. Therefore, the results of the present study should not be considered as absolute values, but rather as relative values to distinguish between the effects of different loading regimes. Using a more sophisticated constitutive model for skeletal muscle tissue (e.g. see Van Loocke et al. ${ }^{29}$ ) would improve the FE model in the sense that a more accurate estimation of threshold values could be obtained. Including friction between the indenter and rat leg strongly influenced and improved the overlap between the outer contours of the leg in FE model and experiment, and also influenced the locations in the FE model where the largest deformations were 
present. Including friction was therefore considered to represent a major improvement to the model.

However, even with the improved FE model, the comparison of deformation and damage in the $2 \mathrm{~h}$ continuous loading regime showed a consistent spatial mismatch between the deformation and damage profiles. This mismatch might be caused by the relative sliding of different muscle groups during loading, which was not incorporated in the FE model. Apparently, this effect did not occur within a 10 min loading period, since almost complete overlap of damage and high deformations was observed for the $10 \mathrm{~min}$ loading period and the $2 \mathrm{~h}$ intermittent loading protocol consisting of 12 periods of $10 \mathrm{~min}$ loading. This spatial mismatch could not affect the global analysis. However, it clearly influenced the results of the local analysis in which a probability function for damage was estimated. Since part of the damage as assessed from the MR images was located in low-strain areas in the FE model instead of the high-strain region, the mismatch probably caused an overestimation of the probability on damage for low strain energy densities, and an underestimation of this probability for high strain energy densities. The results of the local analysis for the $2 \mathrm{~h}$ continuous loading regime should therefore be treated with caution and should not be interpreted as absolute values.

Damage in the tissue was only present if a distinct deformation threshold, of similar magnitude for each of the three loading regimes, was exceeded. This result was obtained from both a global analysis of the results in which the strain energy in the tissue was compared with the total area exhibiting a $T_{2}$ increase, and a local analysis in which the local $\mathrm{T}_{2}$ increase was compared with the local strain energy density. The global analysis showed that no damage occurred for experiments with $2 \mathrm{D}$ strain energies below a threshold range of $0.8 \times 10^{-4}$ to $1.0 \times 10^{-4} \mathrm{~J} / \mathrm{mm}$. The advantage of this analysis is that the deformation and damage in each experiment could be classified with a single value, facilitating the comparison of the different experiments and loading regimes. However, since the derived threshold value is only applicable to the specific deformation pattern applied in this study, it should not be interpreted as an absolute value. ROC curves were used to estimate a local deformation threshold that is effectively independent of the applied deformation profile. Due to the spatial mismatch between deformation and damage for the $2 \mathrm{~h}$ continuous loading regime, the derived cutoff probability for this loading regime was considerably larger than for the other two protocols, which is considered an artifact. The local deformation threshold itself appeared to be similar for each loading regime, corresponding to a strain energy density of approximately 6-7 $\mathrm{kPa}$. It should, however, be noted that the absolute value of this threshold depends on the constitutive model and the values of the material parameters in the FE model. The fact that the deformation threshold for damage within a $2 \mathrm{~h}$ loading period was independent of the load exposure time supports the idea of an inverse sigmoid relation between deformation and time that will lead to tissue damage. ${ }^{13,15,17}$ For short loading periods, the deformation effectively determines the threshold for damage, which corresponds to the initial upper plateau phase of the sigmoid curve. It appears that for the present animal model this initial plateau phase exists for at least $2 \mathrm{~h}$. However, it should be noted that the threshold value and the duration of this initial plateau phase can be different for other animals and in particular for humans, depending on their susceptibility to tissue damage induced by mechanical loading. Thus, an extrapolation of the present threshold to the human situation, particularly with the inherent variability associated with confounding factors, must be taken with caution.

When the deformation threshold for damage was exceeded, the global analysis showed a monotonic increase of the amount of muscle damage with the internal strain energy. This increase of damage with strain energy was larger after a $2 \mathrm{~h}$ loading period than after $10 \mathrm{~min}$. From the local analysis, the probability of damage as a function of the local strain energy density also appeared to be higher for the $2 \mathrm{~h}$ than the 10 min loading period. Thus, the load exposure time influences the amount of damage that develops due to deformation. This increase in affected area in the tissue might be caused by a physiological effect. For example, the release of cellular constituents from dead cells may trigger cell death in the surrounding tissue. Another possible explanation could be the local change of mechanical properties associated with dead tissue. For example, Gefen et al. ${ }^{14}$ found a significant increase in muscle stiffness after cell death, which can subsequently increase the deformations of the surrounding tissue and thereby the total amount of damage.

When the $2 \mathrm{~h}$ continuous and intermittent loading regimes are compared in the global analysis, the increase of damage with strain energy appeared to be similar for both loading regimes. On the other hand, the local analysis suggested distinct differences in the local probability of damage. The continuous loading protocol caused more damage than the intermittent load for small strain energy density values, while the reverse was the case for high strain energy densities. However, it is postulated that these differences in the local analysis were caused by the mismatch between the damage and deformation profiles in the $2 \mathrm{~h}$ continuous loading regime. This mismatch is considered a shortcoming of the FE model, and therefore a 
comparison of these two loading regimes based on the results of the local analysis would lead to erroneous conclusions. For the present study, it is therefore concluded that intermittent load relief did not affect the damage evolution within a $2 \mathrm{~h}$ loading period. This may be a result of the fact that a $2 \mathrm{~h}$ loading period is unlikely to cause any ischemic damage in our animal model. Indeed, it has been shown by Stekelenburg et $a .^{25}$ that $2 \mathrm{~h}$ of complete ischemia as induced by a tourniquet induced no significant damage in the tissue. Therefore, reperfusion during load relief would neither reduce any ischemic damage nor cause additional damage due to ischemia/reperfusion injury. During longer periods of mechanical loading, intermittent load reliefs may show a larger influence on the damage evolution, since then ischemia is expected to play a larger role in the damage process in skeletal muscle tissue. In addition, the off-loading period could also have been too short to affect the evolution of muscle damage. Makhsous et al. ${ }^{19}$ measured the perfusion in the buttocks of human volunteers during a sitting protocol including wheelchair pushups and found that these pushup periods of approximately $30 \mathrm{~s}$ were insufficient to provide complete recovery of tissue perfusion. Coggrave and Rose $^{9}$ also reported that almost 2 min of pressure relief were needed to restore tissue oxygen to basal unloaded levels in spinal cord injury individuals.

In summary, the present study showed that the tissue deformations during mechanical loading determine whether or not skeletal muscle damage develops within a short ( $2 \mathrm{~h}$ in our animal model) loading period. The deformations that were applied in this study are clinically relevant when compared to internal soft tissue strains in paraplegic individuals sitting on a hard surface. ${ }^{18}$ Therefore, to prevent muscle damage that already develops during short periods of mechanical loading, it is important to minimize tissue deformations by means of cushions and mattresses with appropriate stiffness, such that the deformation threshold for damage is not exceeded. If the deformations do exceed the threshold, then the exposure time determines how much damage develops in the tissue. Limiting the exposure time may therefore also help to minimize the amount of muscle damage due to mechanical loading. In the present study, intermittent load relief appeared to have minimal effect on the damage evolution. In clinical practice, relief of continuously applied pressure is often accompanied by a change in posture. It is expected that the resulting redistribution of the mechanical load would inevitably influence the damage evolution and may therefore be more important than temporarily restoring tissue perfusion.

\section{ACKNOWLEDGMENTS}

We gratefully acknowledge Jo Habets and Leonie Niesen for their help with the animal experiments. This research was supported by the Dutch Technology Foundation STW, Applied Science Division of NWO and the Technology Program of the Ministry of Economic Affairs.

\section{OPEN ACCESS}

This article is distributed under the terms of the Creative Commons Attribution Noncommercial License which permits any noncommercial use, distribution, and reproduction in any medium, provided the original author(s) and source are credited.

\section{REFERENCES}

${ }^{1}$ Akobeng, A. K. Understanding diagnostic tests 3: receiver operating characteristic curves. Acta Paediatr. 96(5):644647, 2007.

${ }^{2}$ Black, J., M. Baharestani, J. Cuddigan, B. Dorner, L. Edsberg, D. Langemo, M. E. Posthauer, C. Ratliff, G. Taler, and NPUAP. National pressure ulcer advisory panel's updated pressure ulcer staging system. Dermatol. Nurs. 19(4):343-349, 2007.

${ }^{3}$ Bosboom, E. M. H., C. V. C. Bouten, C. W. J. Oomens, F. P. T. Baaijens, and K. Nicolay. Quantifying pressure sore-related muscle damage using high-resolution MRI. J. Appl. Physiol. 95(6):2235-2240, 2003.

${ }^{4}$ Bosboom, E. M. H., M. K. C. Hesselink, C. W. J. Oomens, C. V. C. Bouten, M. R. Drost, and F. P. T. Baaijens. Passive transverse mechanical properties of skeletal muscle under in vivo compression. J. Biomech. 34(10):1365-1368, 2001.

${ }^{5}$ Bouten, C. V., M. M. Knight, D. A. Lee, and D. L. Bader. Compressive deformation and damage of muscle cell subpopulations in a model system. Ann. Biomed. Eng. 29(2): 153-163, 2001

${ }^{6}$ Breuls, R. G., C. V. Bouten, C. W. Oomens, D. L. Bader, and F. P. Baaijens. Compression induced cell damage in engineered muscle tissue: an in vitro model to study pressure ulcer aetiology. Ann. Biomed. Eng. 31(11):1357-1364, 2003.

${ }^{7}$ Ceelen, K. K., A. Stekelenburg, S. Loerakker, G. J. Strijkers, D. L. Bader, K. Nicolay, F. P. T. Baaijens, and C. W. J. Oomens. Compression-induced damage and internal tissue strains are related. J. Biomech. 41(16):33993404, 2008.

${ }^{8}$ Ceelen, K. K., A. Stekelenburg, J. L. J. Mulders, G. J. Strijkers, F. P. T. Baaijens, K. Nicolay, and C. W. J. Oomens. Validation of a numerical model of skeletal muscle compression with MR tagging: a contribution to pressure ulcer research. J. Biomech. Eng. 130(6):061015, 2008.

${ }^{9}$ Coggrave, M. J.., and L. S. Rose. A specialist seating assessment clinic: changing pressure relief practice. Spinal Cord 41(12):692-695, 2003.

${ }^{10}$ Fleckenstein, J. L. Skeletal muscle evaluated by MRI. In: Encyclopedia of Nuclear Magnetic Resonance. Wiley, 1996. 
${ }^{11}$ Gawlitta, D., W. Li, C. W. Oomens, F. P. Baaijens, D. L. Bader, and C. V. Bouten. The relative contributions of compression and hypoxia to development of muscle tissue damage: an in vitro study. Ann. Biomed. Eng. 35(2):273284, 2007.

${ }^{12}$ Geers, M. G. D., R. de Borst, and W. A. M. Brekelmans. Computing strain fields from discrete displacement fields in 2d solids. Int. J. Solids Struct., 33(29):4293-4307, 1996.

${ }^{13}$ Gefen, A. Reswick and rogers pressure-time curve for pressure ulcer risk. Part 2. Nurs. Stand. 23(46):40-44, 2009.

${ }^{14}$ Gefen, A., N. Gefen, and E. Linder-Ganz. In vivo muscle stiffening under bone compression promotes deep pressure sores. J. Biomech. Eng. 127(3):512-524, 2005.

${ }^{15}$ Gefen, A., B. van Nierop, D. L. Bader, and C. W. Oomens. Strain-time cell-death threshold for skeletal muscle in a tissue-engineered model system for deep tissue injury. J. Biomech. 41(9):2003-2012, 2008.

${ }^{16}$ Hosmer, D. W., and S. Lemeshow. Applied Logistic Regression, 2nd edn. Wiley, 2000.

${ }^{17}$ Linder-Ganz, E., S. Engelberg, M. Scheinowitz, and A. Gefen. Pressure-time cell death threshold for albino rat skeletal muscles as related to pressure sore biomechanics. J. Biomech. 39(14):2725-2732, 2006.

${ }^{18}$ Linder-Ganz, E., N. Shabshin, Y. Itzchak, Z. Yizhar, I. Siev-Ner, and A. Gefen. Strains and stresses in subdermal tissues of the buttocks are greater in paraplegics than in healthy during sitting. J. Biomech. 41(3):567-580, 2007.

${ }^{19}$ Makhsous, M., M. Priebe, J. Bankard, D. Rowles, M. Zeigler, D. Chen, and F. Lin. Measuring tissue perfusion during pressure relief maneuvers: insights into preventing pressure ulcers. J. Spinal Cord Med. 30(5):497-507, 2007.

${ }^{20}$ Miller, G. E., and J. Seale. Lymphatic clearance during compressive loading. Lymphology 14(4):161-166, 1981.
${ }^{21}$ Peirce, S. M., T. C. Skalak, and G. T. Rodeheaver. Ischemia-reperfusion injury in chronic pressure ulcer formation: a skin model in the rat. Wound Repair Regen. 8(1):68-76, 2000.

${ }^{22}$ Rosner, B. Fundamentals of Biostatistics, 6th edn. Thomson Brooks/Cole, 2006.

${ }^{23}$ Stekelenburg, A., C. W. J. Oomens, G. J. Strijkers, L. de Graaf, D. L. Bader, and K. Nicolay. A new MR-compatible loading device to study in-vivo muscle damage development in rats due to compressive loading. Med. Eng. Phys. 28(4):331-338, 2006.

${ }^{24}$ Stekelenburg, A., C. W. J. Oomens, G. J. Strijkers, K. Nicolay, and D. L. Bader. Compression-induced deep tissue injury examined with magnetic resonance imaging and histology. J. Appl. Physiol. 100(6):1946-1954, 2006.

${ }^{25}$ Stekelenburg, A., G. J. Strijkers, H. Parusel, D. L. Bader, K. Nicolay, and C. W. Oomens. Role of ischemia and deformation in the onset of compression-induced deep tissue injury: MRI-based studies in a rat model. J. Appl. Physiol. 102(5):2002-2011, 2007.

${ }^{26}$ Thomas, D. R. Prevention and treatment of pressure ulcers: What works? What doesn't? Cleve. Clin. J. Med. 68(8):704-722, 2001.

${ }^{27}$ Tsuji, S., S. Ichioka, N. Sekiya, and T. Nakatsuka. Analysis of ischemia-reperfusion injury in a microcirculatory model of pressure ulcers. Wound Repair Regen. 13(2):209215, 2005.

${ }^{28}$ Ünal, S., S. Ozmen, Y. DemIr, R. Yavuzer, O. LatIfoglu, K. Atabay, and M. Oguz. The effect of gradually increased blood flow on ischemia-reperfusion injury. Ann. Plast. Surg. 47(4):412-416, 2001.

${ }^{29}$ Van Loocke, M., C. G. Lyons, and C. K. Simms. Viscoelastic properties of passive skeletal muscle in compression: stress-relaxation behaviour and constitutive modelling. J. Biomech. 41(7):1555-1566, 2008. 